\title{
N-ferrocenylmethyl-Derivatives As Spike Glycoprotein Inhibitors Of SARS-CoV-2 Using In Silico Approaches
}

\author{
Zegheb Nadjiba*, Elhafnaoui Lanez, Touhami Lanez \\ University of El Oued, VTRS Laboratory, B.P.789, 39000, El Oued, Algeria
}

Corresponding Author: Zegheb Nadjiba, University of El Oued, VTRS Laboratory, B.P.789, 39000, El Oued, Algeria zegheb-nadjiba@univ-eloued.dz

Running title:

Ferrocenyl methyl-Derivatives As Inhibitors Of SARS-CoV-2

\section{Conflict of Interest}

The authors declare no conflict of interest.

\section{Abstract:}

Background and Objectives: By the end of 2019, a novel human coronavirus outbreak started in Wuhan and spread to the world becoming a global pandemic, patients were diagnosed with severe respiratory syndrome. Studies have shown that SARS-CoV2 interact with angiotensin-converting enzyme 2 (ACE2), its host cell receptor, by its Spike Glycoprotein. The aim of this study is to prevent this interaction by inhibiting Spike glycoprotein. Materials and Methods: The interaction of the Spike Glycoprotein of SARSCOV-2 extracted from protein data bank (PDB Code: 6VSB and 6LZG) with 10 different ferrocene derivatives ligands were investigated by performing docking studies using Autodock Tools 4.2. software. Results: The obtained results showed that N-ferrocenylmethyl-3-nitroaniline was the best inhibitor ligand interacted with both proteins of coronavirus with the free binding energy equal to -5.38 and $-6.65 \mathrm{Kcal} / \mathrm{mol}$ for $6 \mathrm{VSB}$ and 6LZG respectively with binding constant values equal to $8.7 \times 10^{3}$ and $72.72 \times$ $10^{3}$ respectively. Calculations revealed that the dominated mode of interaction for all the studied ligands with COVID-19 was the electrostatic mode via at least one H-bond and more than two hydrophobic Pi-Alkyl Bonds. Conclusion: Generally, the results indicated the existence of strong interactions between ligands and spike glycoprotein which prevent the virus to interact to ACE2 receptors.

Keywords: SARS-COV-2, coronavirus, docking, ferrocenyl-methyl, free binding energy, coronavirus, spike glycoprotein. 


\section{Introduction}

At the end of the year 2019, a novel human coronavirus outbreak started in Wuhan, Hubei Province, China and then subsequently spread to more than 200 other countries becoming a global pandemic.Severe-acute respiratory syndrome coronavirus 2 (SARS-CoV-2) was found to be the viral agent responsible for COVID-19 pneumonia, the resulting illness from infection. On May $4^{\text {th }} 2020$, there are 3,633,833 cases and 251,480 deaths globally ${ }^{1}$, although the actual rates could be higher considering low testing and case identification in some regions.

Presently, there are no commonly agreed on prophylactic or therapeutic treatment options forSARS-CoV-2 and a vaccine is not expected to be developed and disbursed to the wider population for 12-18 months ${ }^{2}$. Current treatment aims to reduce viral load of SARS-CoV2and to improve quality of life. Chloroquine and hydroxychloroquine recently cited as a potential treatment to shorten SARS-CoV-2 disease course ${ }^{3}$, but many side effectswere attributed as gastrointestinal effects including nausea, vomiting, diarrhea, abdominal discomfort as well as the incidence of cardio toxic effects, rhythm disorders and the development of cardiomyopathy in patients with rheumatic diseases, also as severe complication the development of retinopathy ${ }^{4-7}$. Furthermore, ferroquine and Hydroxyferroquine are effective inhibitors of SARS-CoV-1 replication with high selectivity ${ }^{8}$. According to the fact that SARS-CoV-2 has many common features with the coronavirus family and has a phylogenetic similarity $(96 \%)$ to the previous species of SARS-CoV- $1^{9}$, the cited inhibitors may be effective against the novel SARS-CoV-2. Therefore, looking for a new drug targetof SARS-Cov-2 therapy is still more than needed.

Receptor recognition by coronavirus is the first and essential step for entering human cells. The homotrimeric spike glycoprotein (S protein), located on the envelope of theSARS-CoV2 , is responsible for receptor recognition. The $\mathrm{S} 1$ subunit of $\mathrm{S}$ protein,containing receptorbinding domain (RBD), directly interacts with the receptor Angiotensin-Converting Enzyme 2 (ACE2)on the human cell membrane while the S2 subunit facilitates virus-cell fusion and entry.Consequently, interrupting the interaction between S protein and ACE2 is a strategy to inhibit virus entry ${ }^{10,11}$.

In this work, in silico approach using docking studies has been performed to study the interaction of $10 \mathrm{~N}$-ferrocenylmethyl-derivatives with SARS-CoV-2 spike glycoprotein 


\section{Materials and Methods}

Study area: The study was carried out at Laboratory of Valorization and Technology of Sahara Resources (VTRS), University of ElOued, Algeria, in March 2020.Study area:

\section{Methodology:}

All the computational calculations were carried out using AutoDock 4.2 program $^{12}$ and were performed by the PC windows 10 with Intel Core 17 microprocessor, 8 GB memory and 64Bit operating system. All the chemical structures of the $10 \mathrm{~N}$-ferrocenylmethyl-derivatives were optimized using Gaussian 09W program package ${ }^{13}$.

\subsection{Structural Optimization:}

The geometries of the studied ligands were first optimized by molecular mechanics (MM), then they were fully re-optimized by the DFT/ B3LYP method with the 6-311G++ $(\mathrm{d}, \mathrm{p})$ basis set using Gaussian 09W program package ${ }^{13}$. The chemical formulas of ligands are presented in figure 1.

\subsection{Protein selection:}

The X-ray crystal structure of the Prefusion 2019-nCoV spike glycoprotein with a single receptor-binding domain up (PDB ID: 6VSB) (figure 2A) and the novel coronavirus spike receptor-binding domain complexed with its receptor ACE2 (PDB ID: 6LZG) (figure 2B), were obtained from Protein DataBank ${ }^{14}$.

\subsection{Molecular docking studies}

The crystal three dimensional structures of the two proteins (PDB ID: 6LZG) ${ }^{15}$ and (PDB ID: $6 \mathrm{VSB}){ }^{16}$ were selected from protein data bank ${ }^{14}$. the targets receptors were first prepared,all water molecules, ligands and cofactors were deleted and the active site was defined using discovery studio visualize ${ }^{17}$. The PDB file of 6VSB contains 3 chains for Spike glycoprotein, so 2 chains were deleted and only chain A was keptto speed up and simplify calculations. Moreover, the PDB file of 6LZG contains 2 chains A and B where Spike protein (chain B) bound with human receptor ACE2 (chain A). Spike protein was chosen rather than the ACE2 receptor for binding pocket search because ACE2 is expressed in various types of human cells and targeting ACE2 might cause more side effects. 
The PDB files of ligands were saved as PDBQT files after adding the polar hydrogen atoms. Grid boxes were generated using the AutoGrid tool, the grid parameters summarized in Table1.

For docking calculations, Lamarckian genetic algorithms were used. All docking experiments consisted of 10 docking runs with 150 individuals and 2,500,000 energy evaluations. The other parameterswere left to their default values. The best conformation was selected with the lower docking energy ${ }^{18-20}$ and was used in the docking analysis using Protein-Ligand Interaction Profiler (PLIP) ${ }^{21}$.

\section{Results}

At the end of docking runs, diverse binding energies of the ligand were obtained with their respective conformations; the stable conformation, which corresponds to the lowest binding energy, was chosen as the best pose. The binding energy and binding constant Kof the docked structures of all ligands with the two proteins are summarized in Table 2. The magnitude of the calculated binding energy indicates a high binding affinity between proteins and the studied ligands, the binding constant $K$ was calculated using Eq. (1).

$$
\Delta G=-R T \ln K(1)
$$

The results indicate that the ligand $\mathrm{Fc}-3 \mathrm{NO}_{2}$ interacted the best with both studied receptors with binding free energies equal to -6.64 and $-5.38 \mathrm{Kcal} / \mathrm{mol}$ for $6 \mathrm{LZG}$ and $6 \mathrm{VSB}$, respectively.Moreover, the ligand $\mathrm{Fc} 3 \mathrm{NO}_{2}$ represents the highest binding constant values.

The chosen ligand interacts with both receptors via hydrogen bonds (Figure 3) and a hydrophobic bond which is of the type Pi-Alkyl bonds (Figure 4), the bonds are summarized in Table 3. For the others ligands interactions information with 6LZG and 6VSB are summarized in Table 3 and Table 4 respectively.

The results showed that the $\mathrm{Fc}_{3} \mathrm{NO}_{2}$ ligand interacted via 4 hydrogen bonds to Ser50, Thr302, Lys304 and Thr315 amino acids of 6VSB protein (Figure 3a), and via 3 hydrogen bonds to Arg403, Glu406 and Gly496 amino acid of 6LZG protein (Figure 3b).

Docking study shows the formation of 2 Hydrophobic bonds between the chosen ligand (Fc$3 \mathrm{NO}_{2}$ ) and Thr274 and Glu298 amino acids of 6VSB (Figure 4a) and 4 hydrophobic bonds between the ligand and Glu406, Lys417, Tyr495 and Tyr505 (Figure 4b) amino acids of 6LZG.The surface view of the docked conformation with 6VSB (figure 5a) and with 6LZG protein (figure $5 \mathrm{~b}$ ), indicating that ligand $\mathrm{Fc}-3 \mathrm{NO}_{2}$ located between the SARS-CoV2, $\mathrm{S}$ protein and ACE2 which can hinder their interaction. The formed bonds of all the rest of the 
nine studied ligands $\mathrm{Fc}_{2} \mathrm{NO}_{2}, \mathrm{Fc}_{4} \mathrm{NO}_{2}, \mathrm{FcA}, \mathrm{Fc} 3 \mathrm{CN}, \mathrm{Fc}-\mathrm{A} 2 \mathrm{NO}_{2}, 2 \mathrm{FcAd}$, FcAd, FcCy and FcTy with 6LZG are shown in (figures: 6a, 6b, 6c, 6d, 6e, 6f, 6g, 6h and 6i) and with 6VSB in (figures: $6 \mathrm{j}, 6 \mathrm{k}, 61,6 \mathrm{~m}, 6 \mathrm{n}, 6 \mathrm{o}, 6 \mathrm{p}, 6 \mathrm{q}$ and $6 \mathrm{r}$ ), respectively.

\section{Discussion:}

Among the 10 studied compounds, the ligand $\mathrm{Fc}-3 \mathrm{NO}_{2}$ bound with the best affinity to both proteins " 2019-nCoV spike glycoprotein',(PDB ID: 6VSB) ${ }^{15}$ and 'novel coronavirus spike receptor-binding domain complexed with its receptor ACE2',(PDB ID: 6LZG) ${ }^{16}$ via the formation of three hydrogen bonds with the former and four with the later, these bonds were formed with the following aminoacids:Lys417, Gly496 and Tyr505. Moreover, most of the other studied compounds such as $\mathrm{Fc}_{2} \mathrm{NO}_{2}, \mathrm{Fc}_{4} \mathrm{NO}_{2}, \mathrm{FcA}_{2} \mathrm{NO}_{2}, \mathrm{Fc} 3 \mathrm{CN}$ show hydrogen bonds with Lys417, however, other ligands bind to Gly496.

Previous studies show that the SARS-CoV-2 spike glycoprotein contacts the bottom side of the ACE2 small lobe via several amino acids such as Try449, Tyr453, Asn487, Tyr489, Gly496, Thr500, Gly502, Tyr505, Leu455, Phe456, Phe486, Gln493, Asn501, Lys417, Gln474 and Gln $498^{22,23}$, in our case of study the ligand $\mathrm{Fc}_{3} \mathrm{NO}_{2}$ also was binds toSARS-CoV2 spike glycoprotein by interacting with the right amino acids, this finding is with a good agreement with published scientific literatures ${ }^{25,26 .}$.

Furthermore, Hydroxychloroquine (HCQ) and artemisinin (ATN) derivatives have shown a docking score in the range of -7.1 to $-5.5 \mathrm{kcal} \mathrm{mol}^{-1}$, the highest docking score of $-5.5 \mathrm{kcal}$ $\mathrm{mol}^{-1}$ was attributed to the $\mathrm{HCQ}^{24}$. The obtained results revealed that the docking score of $\mathrm{Fc} 3 \mathrm{NO}_{2}$ was equal to $-6.6 \mathrm{kcal} \mathrm{mol}^{-1}$, which indicates that $\mathrm{Fc}_{3} \mathrm{NO}_{2}$ is a better binder than the HCQ.

On the other hand, the obtained molecular docking results show the existence of molecular interaction between $\mathrm{Fc}_{3} \mathrm{NO}_{2}$ and the residues Lys417, Gly496 and Tyr505 which in their turns are bound to the ACE2. Consequently, $\mathrm{Fc}-3 \mathrm{NO}_{2}$ prevents the attachment of SARS-CoV2 RBD and ACE2. Similar to these interactions, other bonds can also disturb the interaction in the middle region of the binding interface between the S Protein and ACE2.

The obtained results can pave the route to the discovery of potentially pharmacochemistry drugs for curing SARS-COV-2 diseases. Furthermore, the results can also be extended to be applied on more ferrocene derivatives and on other types of SARS-COV-2 proteins. Finally, although the obtained in silico results are very promising, in vitro and in vivo experiments should be carried out in order to get more information in relation to the development of the pharmacochemistry activities of the studied derivatives. 


\section{Conclusion:}

A series of ten $\mathrm{N}$-ferrocenyl-methyl-derivatives have been docked successfully, the docking results of N-ferrocenyl-methyl-3-nitroaniline ligand with both COVID-19 proteins (SARS$\mathrm{COV}-2$ ) indicated the existence of strong interactions between ligands and spike glycoprotein which prevent the virus to interact to ACE2 receptors.Fc-3 $3 \mathrm{NO}_{2}$ ligand interact with targets proteins via both H-bonds and hydrophobic Pi-Alkyl where the electrostatic mode was the dominated mode.

\section{Significance Statement:}

This study discovers the high affinity of the interaction between ferrocenylmethyl derivatives and COVID-19 proteins which can be used for in vitro study.

This study will help the researchers to pick up the better ligand candidates for the experimental tests.

\section{Acknowledgements:}

The authors are grateful to the Algerian Ministry of Higher Education and Scientific Research for financial support. We would also like to thank Mr Ali Tliba (VTRS staff) for his help.

\section{Reference:}

1. Worlometer. COVID-19 CORONAVIRUS PANDEMIC. [Available from] https://www.worldometers.info/coronavirus/.

2. Kearney, J., 2020. Chloroquine as a Potential Treatment and Prevention Measure for the 2019 Novel Coronavirus: A Review.DOI: 10.20944/preprints202003.0275.v1

3. Gao, J. Z. Tian, and X. Yang, 2020. Breakthrough: Chloroquine phosphate has shown apparent efficacy in treatment of COVID-19 associated pneumonia in clinical studies. Bio Science Trends. DOI: 10.5582/bst.2020.01047

4. Dogar, M.U.et al, 208. Hydroxychloroquine-induced restrictive cardiomyopathy: a case report.Postgraduate medical journal.,94(1109): p. 185-186. DOI: $10.1136 /$ postgradmedj-2017-135236

5. Chang, I.C. et al, 2017. Hydroxychloroquine-Mediated Cardiotoxicity with a False Positive 99MTechnetium Labeled Pyrophosphate Scan for Amyloidosis.Journal of Cardiac Failure.,23(8): p. S46S47. DOI: 10.1161/CIRCIMAGING.117.007059

6. Chatre, C. et al, 2018. Cardiac complications attributed to chloroquine and hydroxychloroquine: a systematic review of the literature.Drug safety.,41(10): p. 919-931. DOI:10.1007/s40264-018-0689-4

7. Costedoat-Chalumeau, N. et al, 2007. Heart conduction disorders related to antimalarials toxicity: an analysis of electrocardiograms in 85 patients treated with hydroxychloroquine for connective tissue diseases. Rheumatology.,46(5): p. 808-810. DOI: 10.1093/rheumatology/kel402

8. Biot, C. et al, 2006. Design and synthesis of hydroxyferroquine derivatives with antimalarial and antiviral activities.Journal of medicinal chemistry.,49(9): p. 28452849.https://doi.org/10.1021/jm0601856 
9. Zhu, N. et al, 2020. A novel coronavirus from patients with pneumonia in China. New England Journal of Medicine.DOI: 10.1056/NEJMoa2001017

10. Belouzard, S. V.C. Chu, and G.R. Whittaker, 2009. Activation of the SARS coronavirus spike protein via sequential proteolytic cleavage at two distinct sites.Proceedings of the National Academy of Sciences.,106(14): p. 5871-5876.DOI: 10.1073/pnas.0809524106

11. Zumla, A. et al, 2016. Coronaviruses - drug discovery and therapeutic options. Nature reviews Drug discovery.,15(5): p. 327.DOI: $\underline{10.1038 / \mathrm{nrd} .2015 .37}$

12. Morris, G.M. et al, 2009. AutoDock4 and AutoDockTools4: Automated docking with selective receptor flexibility.Journal of computational chemistry., 30(16): p. 2785-2791. DOI: 10.1002/jcc.21256

13. Frisch, M. et al, 2009. Gaussian, Inc., Wallingford CT. Gaussian 09.

14. Berman, H.M. et al, 2002. The protein data bank. Acta Crystallographica Section D: Biological Crystallography.,58(6): p. 899-907. DOI:10.1107/s0907444902003451

15. Wang, Q.H.H. Song, J. X. Qi, 2020. Structure of novel coronavirus spike receptor-binding domain complexed with its receptor ACE2. Protein data bank, 2020.DOI: $\underline{10.2210 / \mathrm{pdb} 6 \mathrm{LZG} / \mathrm{pdb}}$

16. Wrapp, D. et al, 2020. Cryo-EM structure of the 2019-nCoV spike in the prefusion conformation.Science.,367(6483): p. 1260-1263. DOI: $\underline{10.1126 / \text { science.abb2507 }}$

17. Systemes, D, 2016. BIOVIA Discovery Studio Visualizer 2016. San Diego, CA: Dassault Systemes.

18. Darwin, K.H. et al, 2003. The proteasome of Mycobacterium tuberculosis is required for resistance to nitric oxide.Science.,302(5652): p. 1963-1966. DOI: $10.1126 /$ science.1091176

19. Bandyopadhyay, N. et al, 2016.Synthesis, structure, DFT calculations, electrochemistry, fluorescence, DNA binding and molecular docking aspects of a novel oxime based ligand and its palladium (II) complex. Journal of Photochemistry and Photobiology B: Biology.,160: p. 336346.DOI: $10.1016 /$ j.jphotobiol.2016.04.026

20. Guhathakurta, B. et al, 2017. Synthesis, characterization, structure, DNA binding aspects and molecular docking study of a novel Schiff base ligand and its bis ( $\mu$-chloro) bridged $\mathrm{Cu}$ (II) dimer.Polyhedron., 126: p. 195-204.https://doi.org/10.1016/j.poly.2017.01.033

21. Salentin, S. et al, 2015. PLIP: fully automated protein-ligand interaction profiler. Nucleic acids research, 43(W1): p. W443-W447.DOI: 10.1093/nar/gkv315

22. Lan, J. and J. Ge, J. Yu. et al,2020. Structure of the SARS-CoV-2 spike receptor-binding domain bound to the ACE2 receptor. Naturehttps://doi.org/10.1038/s41586-020-2180-5.

23. YAN, Renhong, et al. 2020. Structural basis for the recognition of SARS-CoV-2 by full-length human ACE2. Science., 367.6485: 1444-1448.DOI:10.1126/science.abb2762.

24. SEHAILIA, Moussa, et al, 2020. In-silico Studies of Antimalarial-agent Artemisinin and Derivatives Portray More Potent Binding to Lys353 and Lys31-Binding Hotspots of SARS-CoV-2 Spike Protein than Hydroxychloroquine: Potential Repurposing of Artenimol for COVID-19..

25. Jun L. et al. 2020. Crystal structure of the 2019-nCoV spike receptor-binding domain bound with the ACE2 receptor. BioRxiV. DOI: https://doi.org/10.1101/2020.02.19.956235

26. Bipin, S. 2020. Understanding the role of key point Mutations in Receptor Binding Domain of SARSCoV-2 Spike Glycoprotein. J. life sciences. DOI: 10.20944/preprints202003.0394.v2

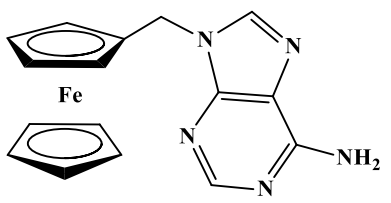

Fc-Ad

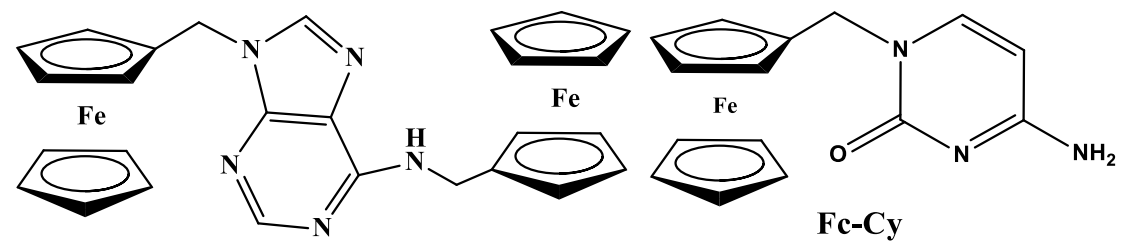

2Fc-Ad (a) (b) (c) 


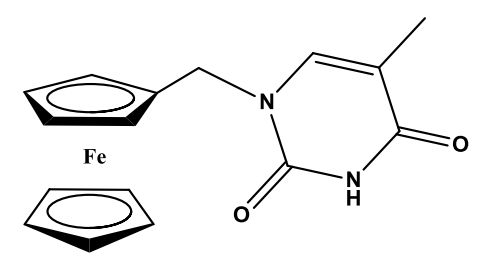

Fc-Ty

(d)

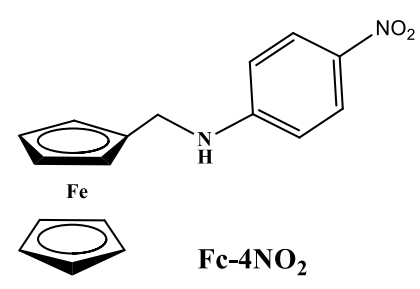

(g)

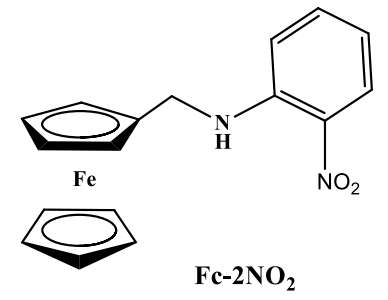

(e)

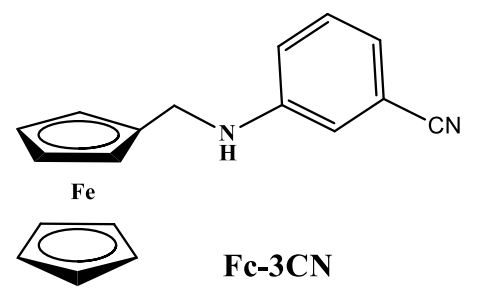

(h)

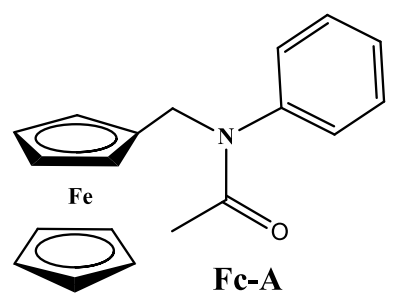

(j)

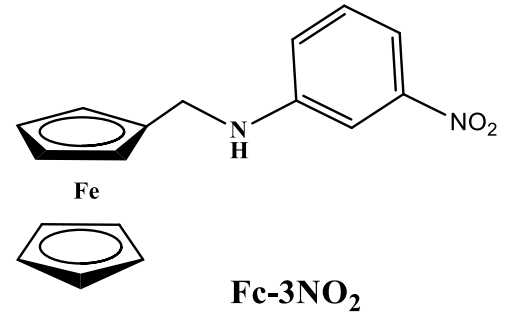

(f)

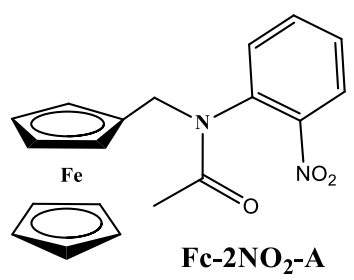

(i)

Fig.1.structure of studied ligands:

(a):ferrocenylmethyl-Adenine,(b): Bis-ferrocenylmethyl-Adenine,(c):ferrocenylmethyl-

Cytosine,(d): ferrocenylmethyl-Thymine,(e): N-(ferrocenylmethyl)-2-nitroaniline,

(f): $\mathrm{N}$-(ferrocenylmethyl)-3-nitroaniline,(g): N-(ferrocenylmethyl)-4-nitroaniline,(h): $\mathrm{N}$ -

(ferrocenylmethyl)-3-aminobenzonitrile, (i): $\mathrm{N}$-(ferrocenylmethyl)-N-acetyl-2-nitroaniline,

(j): N-ferrocenylmethyl-N-phenylacetamide.
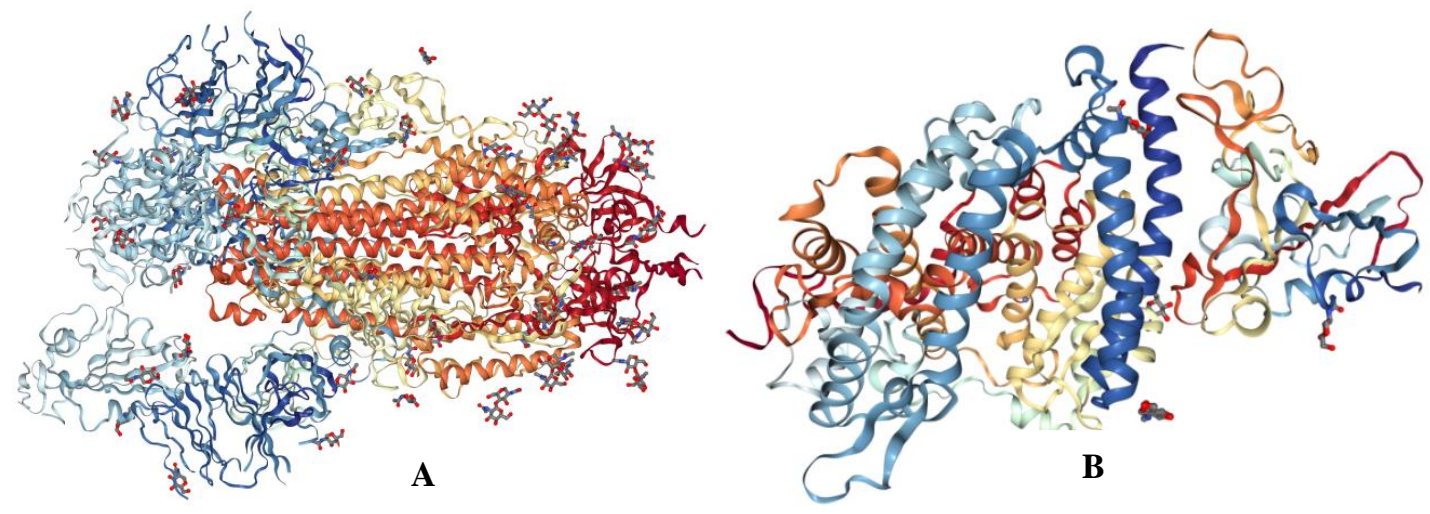

Fig.2. 3D Structures of SARS-CoV-2 spike glycoproteinID: 6VSB (A) and 6LZG.(B) 


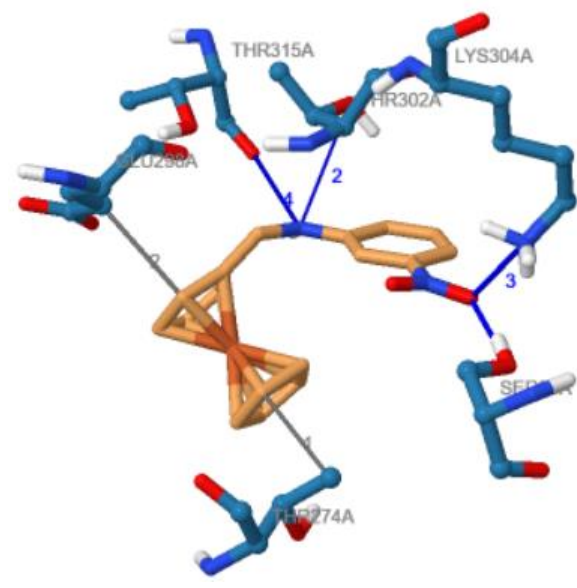

a

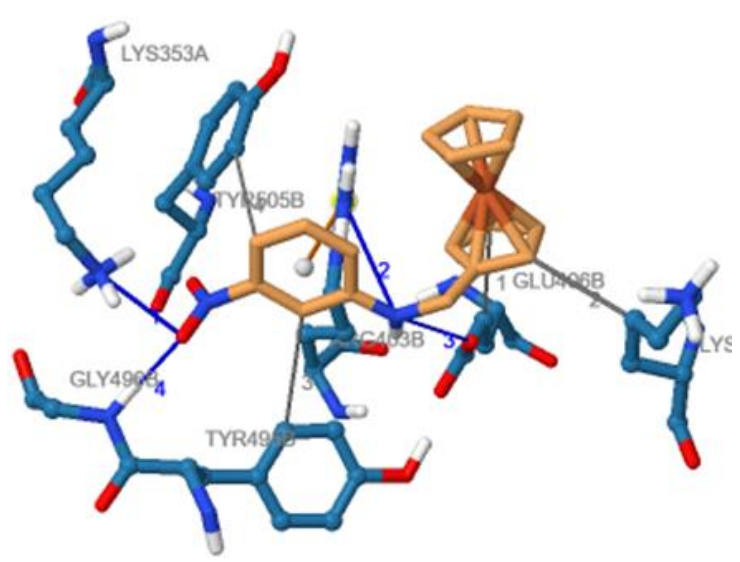

b

Fig.3. 3D interaction between $\mathrm{Fc}_{3} \mathrm{NO}_{2}$ and the target 6VSB (a) and 6LZG(b) where the greylines show hydrophobic bonds and the bleu lines show the H-bonds.

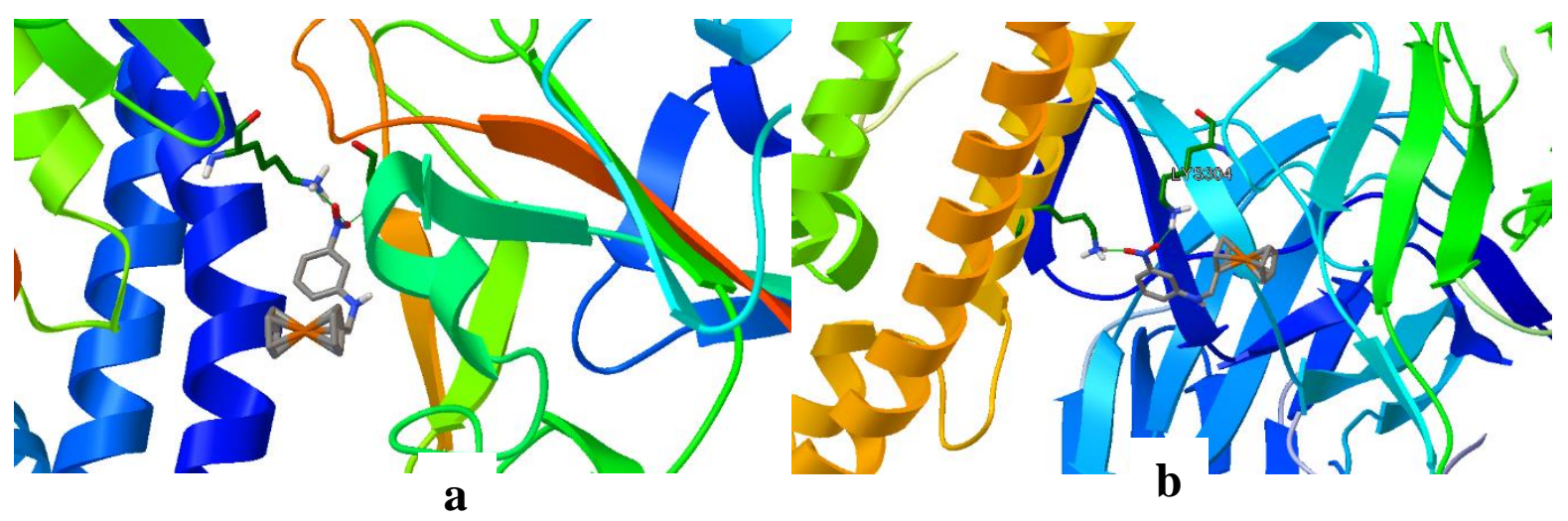

Fig.4.Docking poses of $\mathrm{Fc}_{3} \mathrm{NO}_{2}$ with 6LZG (a) and 6VSB (b) illustrating the interactions between the proteins and the examined ligand.
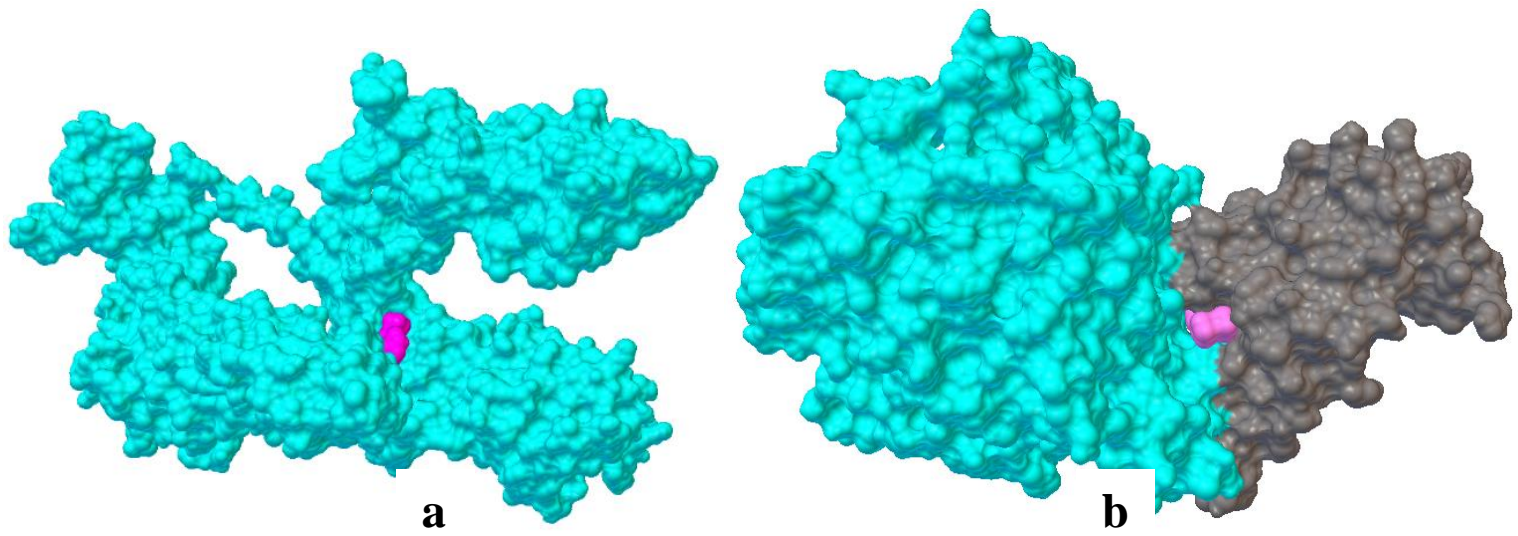
Fig.5.Surface view of docked Fc-3NO 2 with 6VSB (a) and 6LZG (b) where the grey part in (b) presents $\mathrm{S}$ protein.

Table1. Grid parameters used to generate the AutoGrid

\begin{tabular}{|c|c|c|}
\hline Parameters & 6VSB & 6LZG \\
\hline Size $(\AA)$ & $40 \times 40 \times 80$ & $40 \times 60 \times 20$ \\
\hline Spacing $(\AA)$ & 1 & 0.5 \\
\hline & $\mathrm{X}=206.05$ & $\mathrm{X}=-26.793$ \\
Coordinates & $\mathrm{Y}=223.413$ & $\mathrm{Y}=24.94$ \\
& $\mathrm{Z}=215.534$ & $\mathrm{Z}=6.522$ \\
\hline
\end{tabular}

Table 2. Binding free energies and binding constant values obtained by molecular docking approach.

\begin{tabular}{|c|c|c|c|c|}
\hline \multirow{2}{*}{ Compounds } & \multicolumn{2}{|c|}{ 6VSB } & \multicolumn{2}{c|}{ 6LZG } \\
\cline { 2 - 5 } & $\begin{array}{c}-\Delta \mathbf{G} \\
(\mathbf{K c a l} / \mathbf{m o l})\end{array}$ & $\mathbf{K}\left(\mathbf{M}^{-\mathbf{1}}\right)$ & $\begin{array}{c}-\Delta \mathbf{G} \\
(\mathbf{K c a l} / \mathbf{m o l})\end{array}$ & $\mathbf{K}\left(\mathbf{M}^{-\mathbf{1}}\right)$ \\
\hline Fc2NO2 $_{2}$ & -5.02 & $4.7 \times 10^{3}$ & -4.6 & $2.33 \times 10^{3}$ \\
\hline Fc3NO2 $_{2}$ & -5.38 & $8.7 \times 10^{3}$ & -6.64 & $72.72 \times 10^{3}$ \\
\hline Fc4NO2 $_{2}$ & -5.33 & $7.9 \times 10^{3}$ & -6.56 & $63.55 \times 10^{3}$ \\
\hline FcA & -4.15 & $1.1 \times 10^{3}$ & -3.96 & $0.793 \times 10^{3}$ \\
\hline Fc3CN & -4.41 & $1.7 \times 10^{3}$ & -6.12 & $30.267 \times 10^{3}$ \\
\hline Fc2NO2A & -4.98 & $4.4 \times 10^{3}$ & -4.66 & $2.582 \times 10^{3}$ \\
\hline 2FcAd & -4.47 & $1.9 \times 10^{3}$ & -4.18 & $1.149 \times 10^{3}$ \\
\hline FcAd & -4.71 & $2.8 \times 10^{3}$ & -5.76 & $16.496 \times 10^{3}$ \\
\hline FcCy & -3.78 & $0.6 \times 10^{3}$ & -3.78 & $0.585 \times 10^{3}$ \\
\hline FcTy & -4.04 & $0.9 \times 10^{3}$ & -4.80 & $3.269 \times 10^{3}$ \\
\hline
\end{tabular}

$\Delta \mathrm{G}$ : Free binding energy

$\mathrm{K}$ : The binding constant

Table 3. Distances of formed bonds between ligands and 6LZG receptor's residues

\begin{tabular}{|c|c|c|c|}
\hline Adduct & Bond type & AA & Distance \\
\hline \multirow{4}{*}{$\mathrm{Fc} 2 \mathrm{NO}_{2}-6 \mathrm{LZG}$} & \multirow{3}{*}{$\begin{array}{c}\text { Hydrophobic } \\
\text { Pi-Alkyl }\end{array}$} & ASP405B & 3.98 \\
\hline & & GLU406B & 3.20 \\
\hline & & LYS417B & 3.56 \\
\hline & H-bond & ARG403B & 1.94 \\
\hline \multirow{6}{*}{$\mathrm{Fc}_{4} \mathrm{NO}_{2}-6 \mathrm{LZG}$} & \multirow{4}{*}{$\begin{array}{c}\text { Hydrophobic } \\
\text { Pi-Alkyl }\end{array}$} & GLU406B & 3.19 \\
\hline & & LYS417B & 3.34 \\
\hline & & TYR453B & 3.91 \\
\hline & & TYR496B & 3.23 \\
\hline & \multirow{2}{*}{ H-bond } & ARG403B & 2.78 \\
\hline & & GLY496B & 2.43 \\
\hline \multirow{2}{*}{ FcA-6LZG } & \multirow{2}{*}{$\begin{array}{c}\text { Hydrophobic } \\
\text { Pi-Alkyl }\end{array}$} & ASP405B & 3.70 \\
\hline & & GLU406B & 3.21 \\
\hline
\end{tabular}




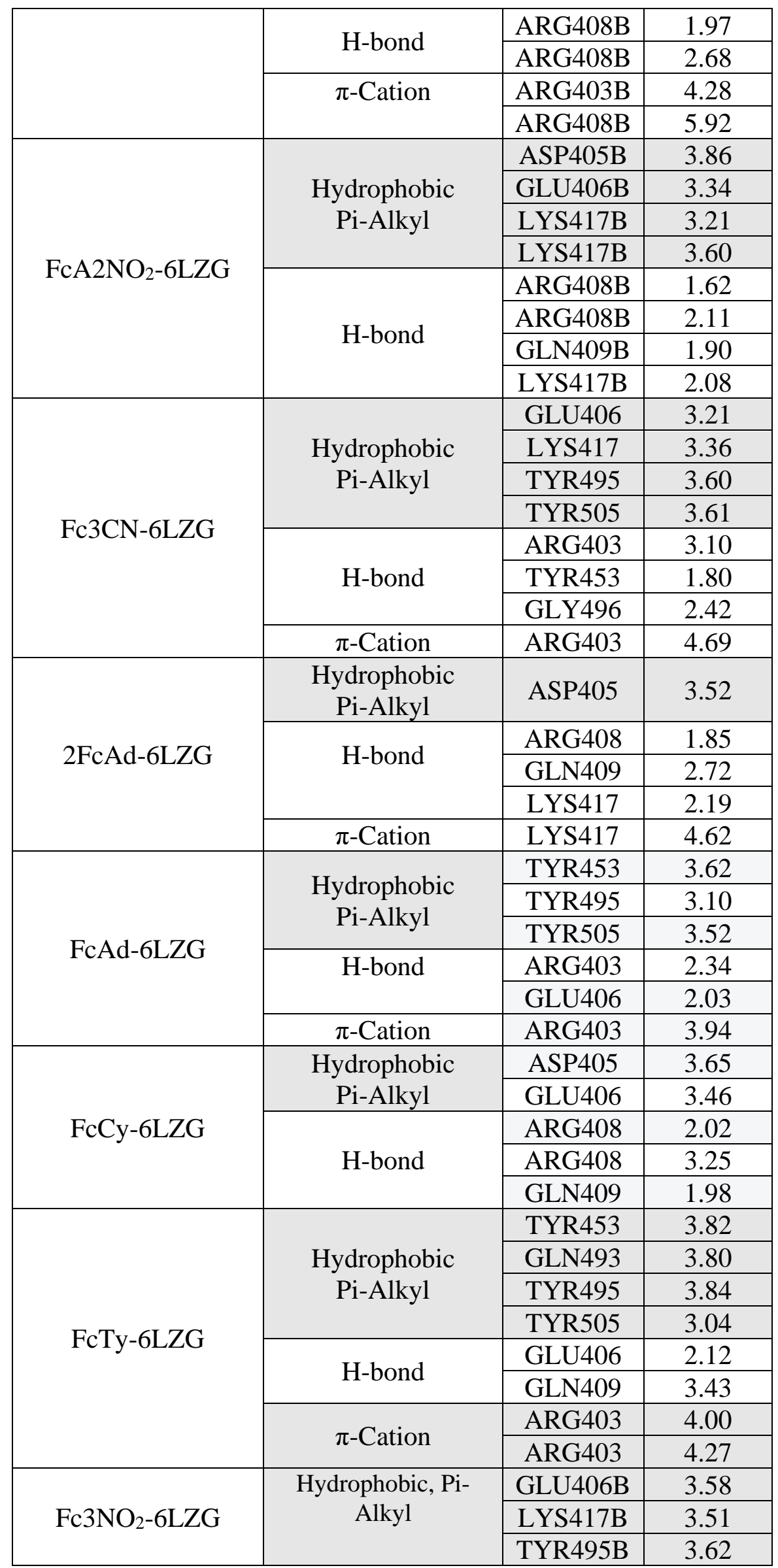




\begin{tabular}{|l|l|l|l|}
\hline \multirow{2}{*}{ H-bond } & TYR505B & 3.95 \\
\cline { 2 - 4 } & & ARG403B & 2.93 \\
\cline { 2 - 4 } & GLU406B & 2.64 \\
\hline
\end{tabular}

Table 4. Distances of formed bonds between ligands and 6VSB receptor's residues

\begin{tabular}{|c|c|c|c|}
\hline Adduct & Bond type & AA & Distance \\
\hline \multirow{3}{*}{ FcAd-6VSB } & $\begin{array}{c}\text { Hydrophobic } \\
\text { Pi-Alkyl }\end{array}$ & THR274A & 3.79 \\
\hline & \multirow{2}{*}{ H-bond } & LYS304A & 2.46 \\
\hline & & LYS964A & 2.04 \\
\hline \multirow{6}{*}{ 2FcAd-6VSB } & \multirow{2}{*}{$\begin{array}{c}\text { Hydrophobic } \\
\text { Pi-Alkyl }\end{array}$} & THR274A & 3.93 \\
\hline & & GLU298A & 3.31 \\
\hline & \multirow{4}{*}{ H-bond } & SER50A & 2.14 \\
\hline & & THR302A & 3.31 \\
\hline & & LYS304A & 1.83 \\
\hline & & THR315A & 2.85 \\
\hline \multirow{5}{*}{ FcCy-6VSB } & \multirow{2}{*}{$\begin{array}{c}\text { Hydrophobic } \\
\text { Pi-Alkyl }\end{array}$} & THR274A & 3.89 \\
\hline & & GLU298A & 3.53 \\
\hline & \multirow{3}{*}{ H-bond } & SER50A & 2.20 \\
\hline & & LYS304A & 1.79 \\
\hline & & THR315A & 2.55 \\
\hline \multirow{3}{*}{ FcTy-6VSB } & $\begin{array}{c}\text { Hydrophobic } \\
\text { Pi-Alkyl }\end{array}$ & GLN52A & 3.95 \\
\hline & \multirow{2}{*}{ H-bond } & SER50A & 2.04 \\
\hline & & LYS304A & 1.94 \\
\hline \multirow{8}{*}{$\mathrm{Fc} 2 \mathrm{NO}_{2}-6 \mathrm{VSB}$} & \multirow{6}{*}{$\begin{array}{c}\text { Hydrophobic } \\
\text { Pi-Alkyl }\end{array}$} & PHE318A & 3.41 \\
\hline & & PHE318A & 3.96 \\
\hline & & VAL595A & 3.21 \\
\hline & & VAL595A & 3.59 \\
\hline & & TYR612A & 3.27 \\
\hline & & VAL620A & 3.70 \\
\hline & \multirow{2}{*}{ H-bond } & GLN321A & 2.37 \\
\hline & & TYR612A & 1.97 \\
\hline \multirow{8}{*}{$\mathrm{Fc}_{4} \mathrm{NO}_{2}-6 \mathrm{VSB}$} & \multirow{5}{*}{$\begin{array}{c}\text { Hydrophobic } \\
\text { Pi-Alkyl }\end{array}$} & GLN271A & 3.67 \\
\hline & & PRO272A & 3.60 \\
\hline & & ARG273A & 3.62 \\
\hline & & THR274A & 3.76 \\
\hline & & GLU298A & 3.85 \\
\hline & \multirow{3}{*}{ H-bond } & PRO272A & 1.95 \\
\hline & & THR274A & 3.04 \\
\hline & & THR274A & 3.17 \\
\hline \multirow{5}{*}{$\mathrm{Fc} 2 \mathrm{NO}_{2}-\mathrm{A}-6 \mathrm{VSB}$} & $\begin{array}{c}\text { Hydrophobic } \\
\text { Pi-Alkyl }\end{array}$ & GLU298A & 3.51 \\
\hline & \multirow{4}{*}{ H-bond } & GLN52A & 2.60 \\
\hline & & PRO272A & 1.88 \\
\hline & & THR274A & 2.40 \\
\hline & & THR274A & 3.21 \\
\hline
\end{tabular}




\begin{tabular}{|c|c|c|c|}
\hline \multirow{2}{*}{ Fc3CN-6VSB } & \multirow{2}{*}{ H-bond } & PRO272A & 2.02 \\
\cline { 3 - 4 } & & THR274A & 2.32 \\
\hline \multirow{3}{*}{ FcA-6VSB } & Hydrophobic & ILE909A & 3.49 \\
\cline { 3 - 4 } & Pi-Alkyl & TYR1047A & 3.75 \\
\cline { 3 - 4 } & H-bond & HIS1048A & 2.13 \\
\hline \multirow{5}{*}{ Fc3NO $_{2}$-6VSB } & \multirow{3}{*}{ Hydrophobic, } & THR274 & 3.93 \\
\cline { 3 - 4 } & Pi-Alkyl & GLU298 & 3.31 \\
\cline { 3 - 4 } & & SER50 & 2.14 \\
\cline { 3 - 4 } & \multirow{2}{*}{ H-bond } & THR302 & 3.31 \\
\cline { 3 - 4 } & & THR304 & 1.83 \\
\hline
\end{tabular}

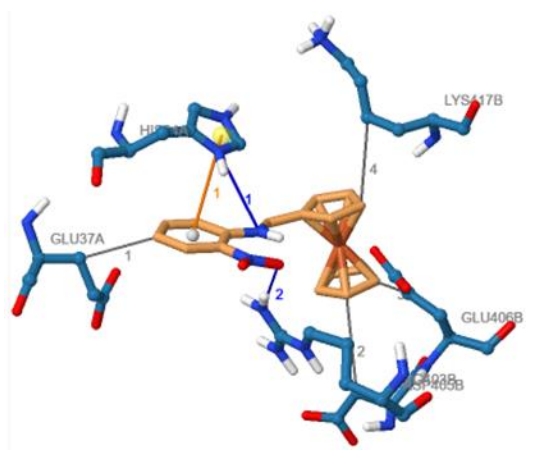

a (Fc2NO2-6LZG)

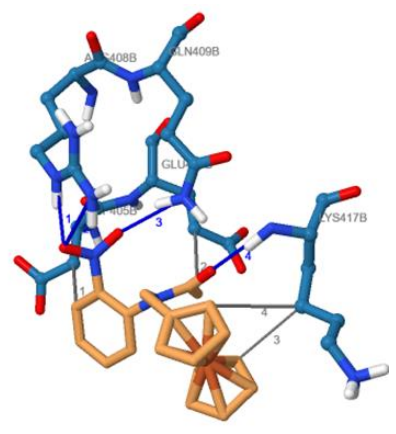

d(Fc2NO2-A-6LZG)

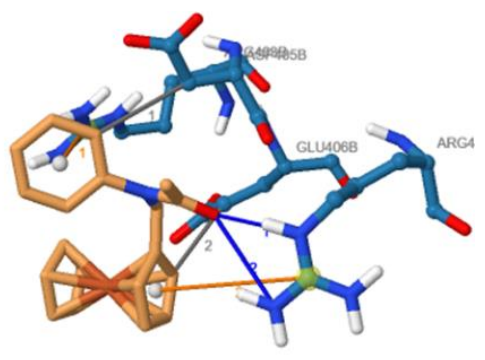

b (FcA-6LZG)

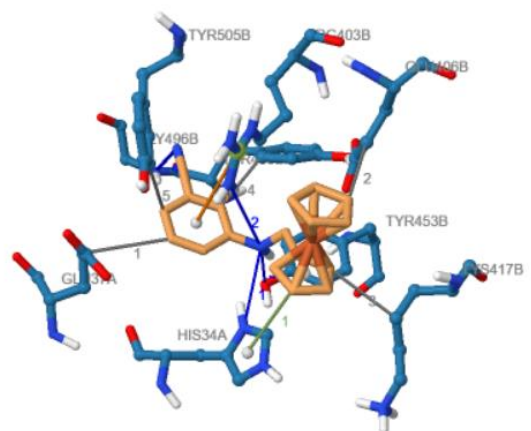

e (Fc3CN-6LZG)

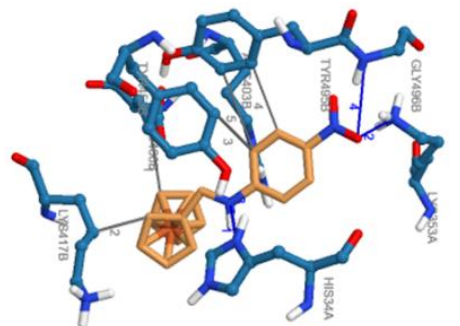

c (Fc4NO2-6LZG)

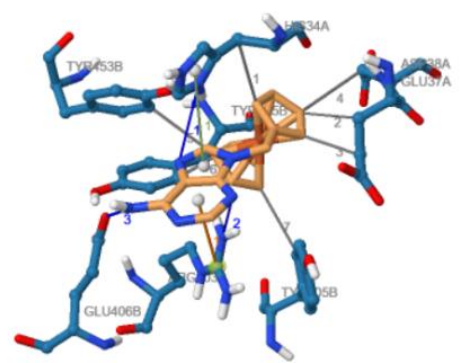

f (FcAd-6LZG) 

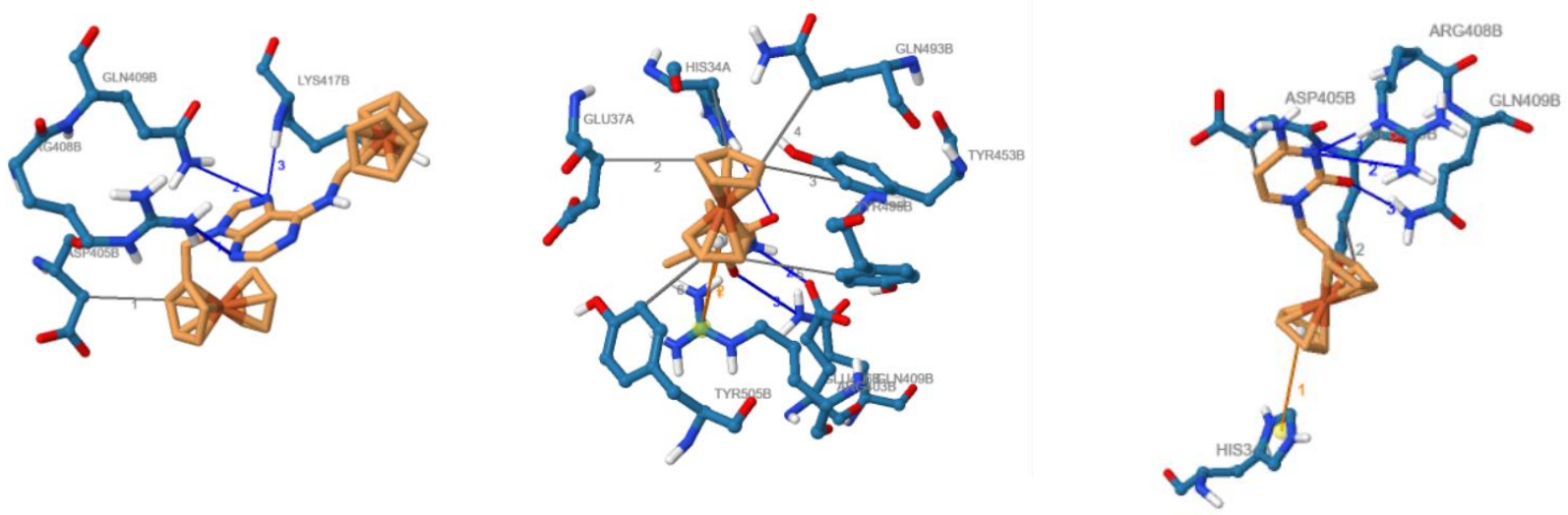

g(2FcAd-6LZG)

h(FcTy-6LZG)

i(FcCy-6LZG)
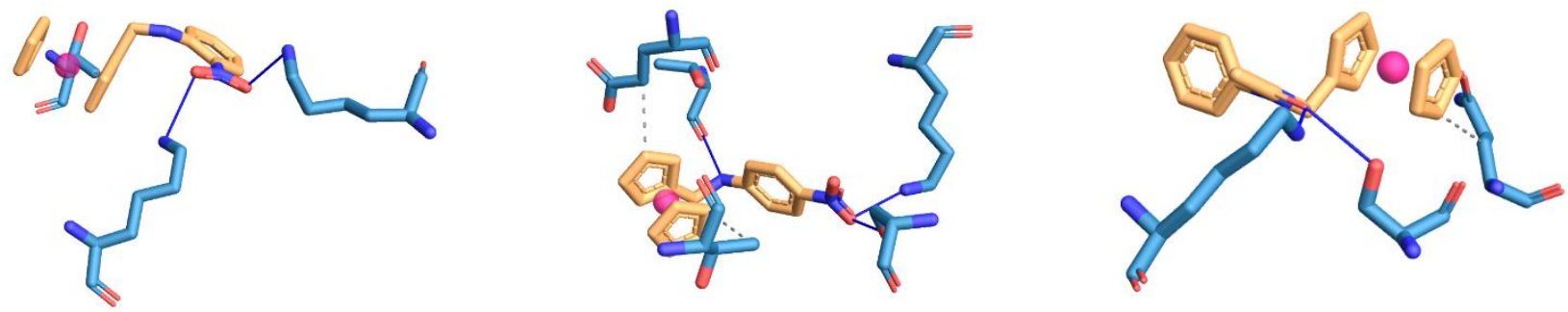

j(Fc2NO2-6VSB $)$

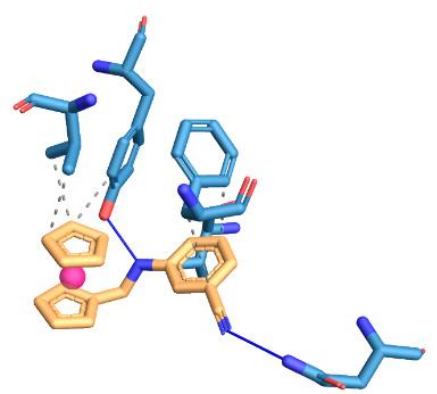

k(Fc4NO2-6VSB)

I(FcA-6VSB)

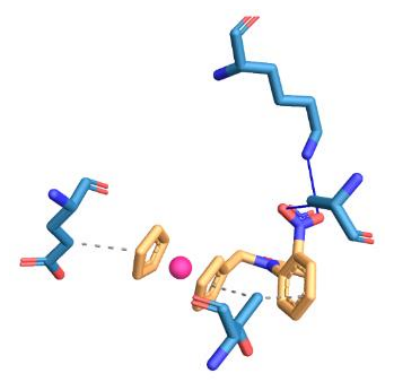

n(Fc2NO2-A-6VSB)

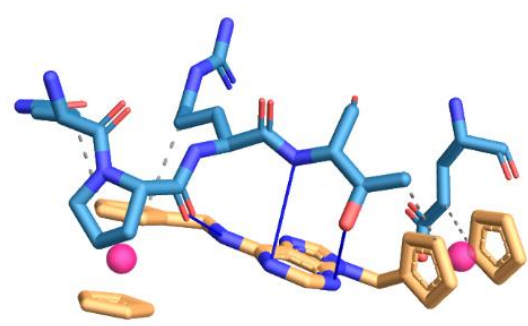

m(Fc3CN-6VSB $)$
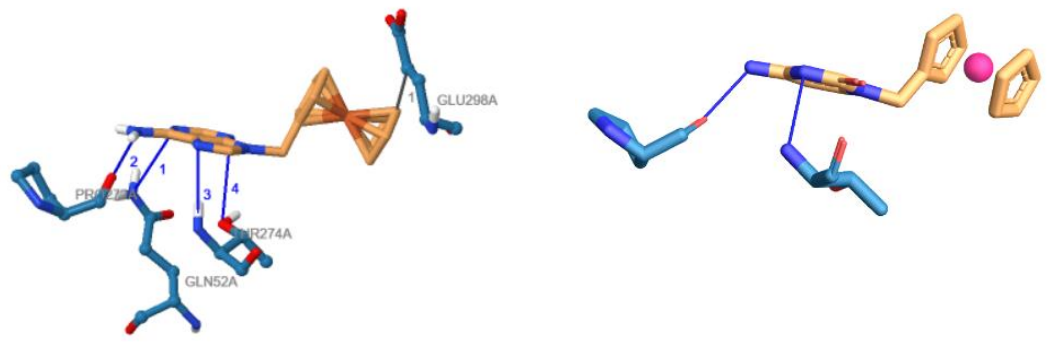

p(FcAd-6VSB)

q(FcCy-6VSB)

r(FcTy-6VSB)

Fig.6.3D interaction between the studied ligands and the targets 6VSB and 6LZG where the greylines show hydrophobic bonds and the bleu lines shows the H-bonds. 